\title{
Haptic Stabilization of Posture in Adults With Intellectual Disabilities Using a Nonrigid Tool
}

\author{
Eliane Mauerberg-deCastro, Carmila Souza Lucena, \\ Bruna W. Cuba, and Rosana C. Boni \\ São Paulo State University
}

\section{Debra Frances Campbell}

University of Alberta

\author{
Renato Moraes \\ University of São Paulo
}

\begin{abstract}
This study assessed the effects of haptic information on the postural control systems of individuals with intellectual disabilities (ID), through the use of a nonrigid tool that we call the "anchor system" (e.g., ropes attached to graduated weights that rest on the floor). Eleven participants with ID were asked to stand, blindfolded, on a balance beam placed at two heights $(10$ and $20 \mathrm{~cm})$, for $30 \mathrm{~s}$, while using the anchor system at two weights. The lighter anchor weight appeared to improve the individuals' balance in contrast to a control task condition; therefore, we concluded that haptic sensitivity was more significant in helping to orient the body than was the anchor's mechanical support alone.
\end{abstract}

Individuals with intellectual disability (ID) invariably exhibit poor or delayed postural adjustments at early stages; and, while many eventually acquire most fundamental motor milestones (e.g., upright position, walking, and running), they continue to experience balance problems as they age (Mauerberg-deCastro \& Kinzler, 2000). Individuals with ID, such as Down syndrome, are known to have slower reactions to perturbations to posture, regardless of their ages (Shumway-Cook \& Woollacott, 1985). Carvalho and Almeida (2009) demonstrated that individuals with Down syndrome adopted a pattern of cocontraction in their leg muscles, making them unable to modulate the magnitude of postural response to a task with varied degrees of instability. This finding reflects a proprioceptive deficit for this group. In addition, as individuals with ID age, lifestyle and health-related problems also contribute to postural instability and often increase the risk of falls and of loss

Eliane Mauerberg-deCastro, Camila de Souza Lucena, Bruna W. Cuba, and Rosana C. Boni are with the Department of Physical Education at São Paulo State University in Rio Claro, Brazil. Debra Frances Campbell is with the Faculty of Physical Education \& Recreation, University of Alberta, Canada. Renato Moraes is with the School of Physical Education and Sport at Ribeirao Preto, University of São Paulo, Brazil. 
of independence (Hale, Bray, \& Littmann, 2007). Lahtinen, Rintala, and Malin (2007), reporting a 30-year longitudinal study of individuals with ID, found that in adolescence, $25 \%$ of the ID participants were able to complete a static balance task (standing on one foot for 60 s, i.e., stork stand test); in adulthood the total of those who could perform the task dropped to less than $10 \%$.

Researchers have focused on causes for poor balance skills found in adults with ID, as well as on the adaptation process underlying postural control. Knowledge about the role of sensory information on postural control in groups that are known to have poor balance, for example, has added to our understanding of how, under a perception-action approach, sensory-motor mechanisms function (Gomes \& Barela, 2007; Jeka, Easton, Bentzen, \& Lackner, 1996; Prioli, Cardozo, Freitas Junior, \& Barela, 2006). Numerous researchers have investigated how postural sway can be attenuated through an individual's acquisition of additional sensory information (somatosensory or visual; Jeka \& Lackner, 1994; Jeka et al., 1996) or by the introduction of task demands to a challenged postural system (Prioli et al., 2006). Sources of postural disruptions can be task-manipulated (i.e., unexpected surface displacement, blindfolding of the participant, etc.), but also can originate intrinsically (i.e., the presence of developmental disabilities or degenerative conditions). Postural instability has been investigated from a perception-action approach relative to the role sensory cues play on it in older individuals (Prioli et al., 2006), in congenitally blind people (Easton, Greene, DiZio, \& Lackner, 1998; Jeka et al., 1996), and in individuals with Down syndrome (Gomes \& Barela, 2007).

Several systems - the visual, vestibular, proprioceptive, and haptic-take part in the evolution of the postural system. In early studies of haptic sensitivity, ${ }^{1}$ researchers focused on the acquisition of information through the exploratory actions of the hands (Cooper, Carello, \& Turvey, 2000; Solomon \& Turvey, 1988). The haptic system's effect on postural control later gained the interest of researchers in the action-perception area (Burton, 1993; Carello, Silva, Kinsella-Shaw, \& Turvey, 2008; Holden, Ventura, \& Lackner, 1994; Lackner \& DiZio, 2005; Rabin, DiZio, \& Lackner, 2006; Rabin, DiZio, Ventura, \& Lackner, 2008). Individuals' use of tools for postural orientation during the maintenance of an upright position, or during the execution of movements such as the handling of objects, can provide us with excellent examples of how humans detect information by exploring different textures and dimensions indirectly, rather than via "biological detectors" such as hands or fingertips.

Numerous researchers have confirmed that the act of merely touching a surface lightly with a fingertip can help individuals stabilize their posture (Johannsen, Wing, \& Hatzitaki, 2007; Lackner \& DiZio, 2005; Lackner, Rabin, \& DiZio, 2001; Rabin, Dizio, \& Lackner, 2006; Rabin, DiZio, Ventura, \& Lackner, 2008). A major finding in this regard is that a force as light as ?0.4 N (applied by the index finger to a stationary surface) can attenuate sway by as much as $60 \%$ (Rabin, Dizio, \& Lackner, 2006). Because mechanical stabilization is not always possible, individuals also rely on haptic sensitivity to detect the direction of body sway (Jeka, Schöner, Dijkstra, Ribeiro, \& Lackner, 1997; Krishnamoorthy, Slijper, \& Latash, 2002).

By employing postural tasks that use the subtle resistance of a nonrigid tool, Mauerberg-deCastro (2004) explored how the body can dynamically detect physical invariants that convey information during the maintenance of the upright position. She used an "anchor" metaphor ${ }^{2}$ to illustrate a mechanism by which individuals 
explore the environment to control the forces that act upon their postural system (Mauerberg-deCastro, 2004): a mechanism that relies on haptic perception. Often, a final goal of an exploratory system's dynamic information pickup is to keep itself still or oriented. The anchor-extension conducts such information via haptic perception about the status of the adjacent surface and at the same time, about perturbations along the extension.

There are two significant differences between the "anchor system" paradigm and the "light touch" paradigm. The first involves the direction of forces relative to gravitational pull. The anchor system employs an upward force; light touch employs a downward force. The second is that, with the anchor system, information about the support surface is mediated through the hands' contact with the implement or "tool" (ropes) and the actual source of information-gathering contact (weights), rather than through direct light contact with a support surface (light touch).

In 2004, the first study using the anchor paradigm indicated that the blindfolded adults appeared to improve in their stability while performing a 30-s, one-foot stance task. Although participants exhibited varied levels of stability, improvement was clearly superior in the anchor conditions as compared with the control condition.

Because the use of the anchor system requires a voluntary process, and because it is embedded in a postural task, it seems relevant to study whether haptic sensitivity in these individuals is impaired, because it relies on decision-making mechanisms. Do individuals with intellectual disabilities successfully use haptic information acquired through nonrigid tools such as the anchor system to reduce postural instability, as has previously been found for adults and children without disabilities? From earlier studies, we found that the anchor system provides greater stability for individuals who were more affected by the postural task (MauerbergdeCastro, 2004; Mauerberg-deCastro, Calve, Viveiros, Polanczyk, \& Cozzani, 2003). Furthermore, in these studies, because no difference between heavy and light anchor weights were found, haptic sensory information mediated by the anchor devices was claimed to be the main factor that affected the stability of the participants. Therefore, we concluded that the mechanical support that originated from the gravitational pull from the weights resting on the floor was a secondary factor in the postural task.

The purpose of this study, then, was to confirm whether the use of a nonrigid tool (i.e., an anchor system) could help adults with intellectual disabilities, who were challenged by visual (i.e., blindfolded) and task constraints (i.e., different support surface heights) during a postural task, achieve stability. It also was to verify, through the use of varying weight resistance, whether sensory haptic cues received during the "anchoring" process_rather than the anchors' mechanical resistance alone-was a primary source of information during the achievement of stabilization. The main assumption of this study is that participants' attempts to reach stability through exploratory motions while using the anchor system do not demand complex decisions based on the deliberate calibration of body segment motions; therefore, individuals at various developmental levels can use it. Our first prediction was that intellectual disability does not preclude the efficient use of the anchor system as an aid in challenging postural tasks, and, therefore, we expected to find significant effect in the balance task condition. Another prediction was that the mechanical support provided by the anchor system would be secondary to the sensory information gathered through it in improving the participant's stability and, 
therefore, we expected to find no significant differences between the two anchor task conditions. Finally, we predicted that the use of the anchor system would be more efficient as the balance task increased the challenge to posture, and, therefore, we expected to find a significant effect relative to the balance beam height.

\section{Method}

\section{Participants}

Eleven adults (three females; eight males) with moderate and mild intellectual disabilities (ID), aged 25.6 years $( \pm 4.7)$, who are participants in a program at a local institution for individuals with intellectual disabilities, were invited to take part in the study. Table 1 summarizes information about the individuals' gender, age, anthropometric data, ID level, and cause for ID. All of the participants were Brazilians, each with multiethnic backgrounds, and none were identified with any specific ethnic group. Each participant was of lower socioeconomic status and without other related known disabilities. With the exception of participant SEM, who had completed the first grade, all of the other participants had a preschool education. At the time of their participation in this study, all were attending vocational skills classes. Legal guardians signed a permission form designed specifically for this study. The Ethics Committee at the São Paulo State University approved the procedures used in the current study.

Table 1 Information About the Individuals' Gender, Age, Anthropometric Data, ID Level, and Cause for ID for Each Participant

\begin{tabular}{lcccclcl}
\hline Participant & Gender & Age & $\begin{array}{c}\text { Weight } \\
(\mathbf{k g})\end{array}$ & $\begin{array}{c}\text { Height } \\
(\mathbf{m})\end{array}$ & ID level $^{*}$ & IQ & Etiology \\
\hline SEM & F & 23 & 92 & 1.61 & Mild & 50 & Unknown \\
CAS & F & 27 & 58 & 1.63 & Mild & 45 & Unknown \\
SIL & F & 23 & 47 & 1.52 & Mild & 45 & Unknown \\
MCB & M & 22 & 67 & 1.65 & Moderate & 40 & Unknown \\
GEN & M & 20 & 118 & 1.66 & Mild & 45 & Neonatal \\
& & & & & & & anoxia \\
CLA & M & 33 & 83 & 1.78 & Moderate & 45 & Unknown \\
LUC & M & 31 & 57 & 1.66 & Moderate & 40 & Unknown \\
RAF & M & 21 & 51 & 1.70 & Mild & 45 & Unknown \\
PAI & M & 24 & 58 & 1.47 & Moderate & 45 & Down \\
& & & & & & & Syndrome \\
JOS & M & 25 & 106 & 1.65 & Mild & 45 & Unknown \\
Mean & & $\mathbf{2 5 . 6}$ & $\mathbf{7 7 . 7}$ & $\mathbf{1 . 6}$ & & 44.1 & \\
SD & & $\mathbf{4 . 7}$ & $\mathbf{2 6 . 9}$ & $\mathbf{0 . 1}$ & & 3.0 & \\
\hline
\end{tabular}

* IQ scores estimated from percentiles of Raven's Colored Progressive Matrices. 


\section{Procedure}

The "anchors" consisted of two pairs of weights: $125 \mathrm{~g}$ and $500 \mathrm{~g}$ for each of the two weights per pair. Each weight consisted of a small cloth bag containing lead pellets, which was connected at one end to a nylon rope. Participants held the opposite ends of the ropes, one in each hand, at approximately waist-height, with the two anchors placed on the floor, one on each side of his or her body. The participants stood blindfolded and were asked, while keeping the anchor ropes stretched and the two anchors resting on the floor, to maintain balance for $30 \mathrm{~s}$ on a support beam, which was placed at two heights, $10 \mathrm{~cm}$ and $20 \mathrm{~cm}$ (Figure 1). We instructed the participants to practice using the anchors as an aid to maintaining balance, but not to lift the weights off the floor. We used simple instructions, such as "Leave the bags on the floor, but, with your hands, pull on the ropes enough to tighten them so they are straight like sticks." The conditions that combined beam height and anchor weight were tested in separate blocks. In each block, participants performed three trials. The order of these blocks was randomized. Participants rested for 30 $\mathrm{s}$ between trials and for 2-min periods between blocks of trials. We granted longer rest periods when participants requested. The duration of each experimental session was less than one hour.

All participants were videotaped with two video cameras (Panasonic M9000) placed perpendicularly to the task scene. Cameras were synchronized by recording a triggered light pulse. The sampling rate was set at $30 \mathrm{~Hz}$. The participants' joints were marked at fifth metatarsal head, lateral malleolus (ankle), lateral epicondyle of the femur (knee), greater trochanter (hip), and greater tubercle of humerus (shoulder), and on both sides of the body. The left side was used for data digitizing.

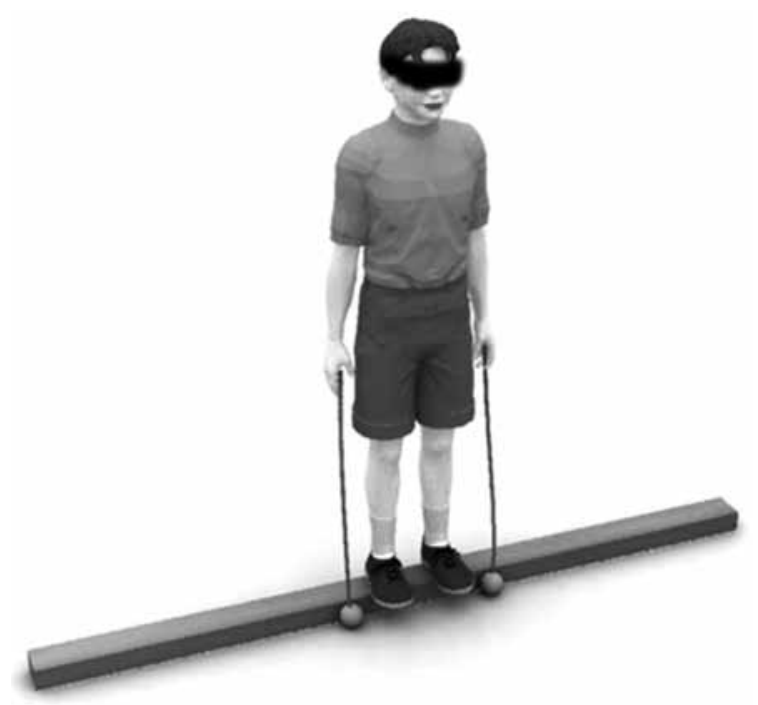

Figure 1 - Task layout using the "anchor system," for the low height beam. 


\section{Data Analyses}

We processed the video data using an integrated video analog-to-digital capturing interface, and then the markers' trajectories were digitized and reconstructed in three-dimensional space using the software DVideow 5.1 (Barros et al., 1997). After residual analysis of the digitized markers' coordinates, we used a fourth-order, zerolag, low-pass digital Butterworth filter, with a cut-off frequency of $6 \mathrm{~Hz}$. Based on the filtered coordinates of $\mathrm{x}, \mathrm{y}$, and $\mathrm{z}$ of the anatomical points, we calculated the center of mass (COM), represented by the three segments monitored: leg, thigh, and trunk. Anthropometric parameters were based on those used by Winter (2005). For trunk center of mass calculation, half trunk mass was used, because markers were placed on only one side of the body.

Based on COM coordinates, the following variables were computed in the anterior-posterior (AP), vertical (V), and medial-lateral (ML) directions: mean sway amplitude (MSA), mean sway velocity (MSV), range of motion (ROM), and peak velocity $(\mathrm{PV})$. Sway amplitude within a trial was computed by subtracting the average position of COM from each data point. MSA was calculated as the standard deviation of the sway amplitude, indicating how dispersed the data were relative to the mean COM position. MSV was calculated by differentiating the sway amplitude and calculating the average velocity within a trial. For the ROM, the maximum and minimum excursions of the COM were obtained within a trial. ROM was computed by calculating the difference between maximum and minimum values. We assumed that task manipulations would increase values in these parameters, representing a more unstable system. For the PV, COM position was differentiated and the peak value of the velocity was obtained within a trial. PV indicates ballistic reactions to momentary loss of balance. In addition, a variable that incorporates modifications in the COM in all three directions was calculated as the distance covered by the COM within a trial (Vuillerme, Isableu, \& Nougier, 2006). We called this variable "COM path length." This measure corresponds to the sum of the distance covered between each consecutive pair of data points within a trial. We also computed trial duration for each participant in each condition. These variables were calculated for only one (randomly) selected block trial.

\section{Statistical Analyses}

For each dependent variable (i.e., MSA, MSV, ROM, PV, trial duration, and path length), two-way ANOVAs (beam height $[10$ and $20 \mathrm{~cm}] \times$ postural task condition [NW, $125 \mathrm{~g}$, and $500 \mathrm{~g}]$ ) with repeated measures for both factors were carried out. Except for the variables' path length and trial duration, these analyses were conducted separately for each direction of motion (AP, V, and ML), totaling fourteen ANOVAs. When ANOVA identified main effect, we carried out Bonferroni post hoc analysis to identify where the differences resided. Bonferroni post hoc analysis uses $t$ tests for pairwise comparisons and automatically adjusts the $p$-level for the number of comparisons to avoid Type I error. When main or interaction effects resulted in significant differences, we computed the effect size using the eta squared $\left(\eta^{2}\right)$ parameter. According to Thalheimer and Cook (2002), an effect size of 0.8 is large, 0.5 is medium or moderate, and 0.2 is small. $P$-value was set at 0.05 for all statistical analyses. 


\section{Results}

All of the participants were able to perform the tasks of all postural task conditions at both balance beam heights. All participants fell off of the balance beam at least once. For the lower balance beam, group average time on each task condition was: $29.3 \mathrm{~s}( \pm 1.6)$ for NW, $29.2 \mathrm{~s}( \pm 1.7)$ for anchor $125 \mathrm{~g}$, and $29.5 \mathrm{~s}( \pm 1.2)$ for anchor $500 \mathrm{~g}$. For the high balance beam, values were: $28.9 \mathrm{~s}( \pm 1.8)$ for NW, $28.6 \mathrm{~s}( \pm$ $3.2)$ for anchor $125 \mathrm{~g}$, and $29.1 \mathrm{~s}( \pm 2.1)$ for anchor $500 \mathrm{~g}$. The two-way ANOVA for trial duration identified no main effects for beam height, $F(1,10)=0.803, p$ $=0.391$ nor postural task condition, $F(2,20)=0.223, p=0.802$. In addition, no interaction effect was found for beam height by postural task condition, $F(2,20)$ $=0.023, p=0.977$.

The ANOVA calculated for the MSA at the AP direction revealed no main effects for beam height, $F(1,10)=4.297, p=0.065$ and postural task condition, $F(2,20)=1.682, p=0.211$ or interaction effect between beam height and postural task condition, $F(2,20)=1.328, p=0.287$. ANOVA for the $\mathrm{V}$ direction revealed no main effect for postural task condition, $F(2,20)=1.320, p=0.290$ and no interaction effect between beam height and postural task condition, $F(2,20)=1.400, p=$ 0.270 , but it identified a main effect for beam height, $F(1,10)=11.189, p=0.007$, $\eta^{2}=0.528$. MSA was higher for the $20 \mathrm{~cm}$ beam than for the $10 \mathrm{~cm}$ beam (Table 2). For the ML direction, ANOVA exhibited no main effect for beam height, $F(1,10)$ $=4.570, p=0.058$ and no interaction effect between beam height and postural task condition, $F(2,20)=0.099, p=0.906$, but it identified a main effect for postural task condition, $F(2,20)=3.682, p=.044, \eta^{2}=0.269$. Post hoc analysis failed to identify pairwise differences for postural task conditions, however.

ANOVA for the MSV in the AP direction identified a main effect for postural task condition, $F(2,20)=7.386, p=.004, \eta^{2}=0.425$, but no main effect for beam height, $F(1,10)=1.431, p=0.259$ and no interaction effect between beam height and postural task condition, $F(2,20)=1.412, p=0.267$. Post hoc analysis identified differences only between NW and $125 \mathrm{~g}$ conditions $(p=0.032)$. The use of the $125 \mathrm{~g}$ anchor reduced MSV as compared with the NW condition. For the $\mathrm{V}$ direction, ANOVA failed to identify main effects for beam height, $F(1,10)=$ $4.198, p=0.068$ and for postural task condition, $F(2,20)=1.155, p=0.335$ and also for the interaction between beam height and postural task condition, $F(2,20)$ $=1.106, p=0.350$. ANOVA for the ML direction did not exhibit main effects for beam height, $F(1,10)=3.684, p=0.084$ and for postural task condition, $F(2,20)$ $=2.591, p=0.100$ nor for the interaction between beam height and postural task condition, $F(2,20)=0.316, p=0.732$.

\section{Range of Motion (ROM) and Peak Velocity (PV)}

ANOVA for ROM detected a main effect for beam height in the AP direction, $F(1$, $10)=5.293, p=0.044, \eta^{2}=0.346$, but no main effect for postural task condition, $F(2,20)=2.511, p=0.106$ and for the interaction between beam height and postural task condition, $F(2,20)=0.784, p=0.470$. For the $\mathrm{V}$ direction, ANOVA identified the same result: beam height, $F(1,10)=16.245, p=0.002, \eta^{2}=0.619$; postural task condition, $F(2,20)=0.835, p=0.448$; beam height by postural task condition, $F(2,20)=0.839, p=0.447$. The beam height main effect for ROM in both 


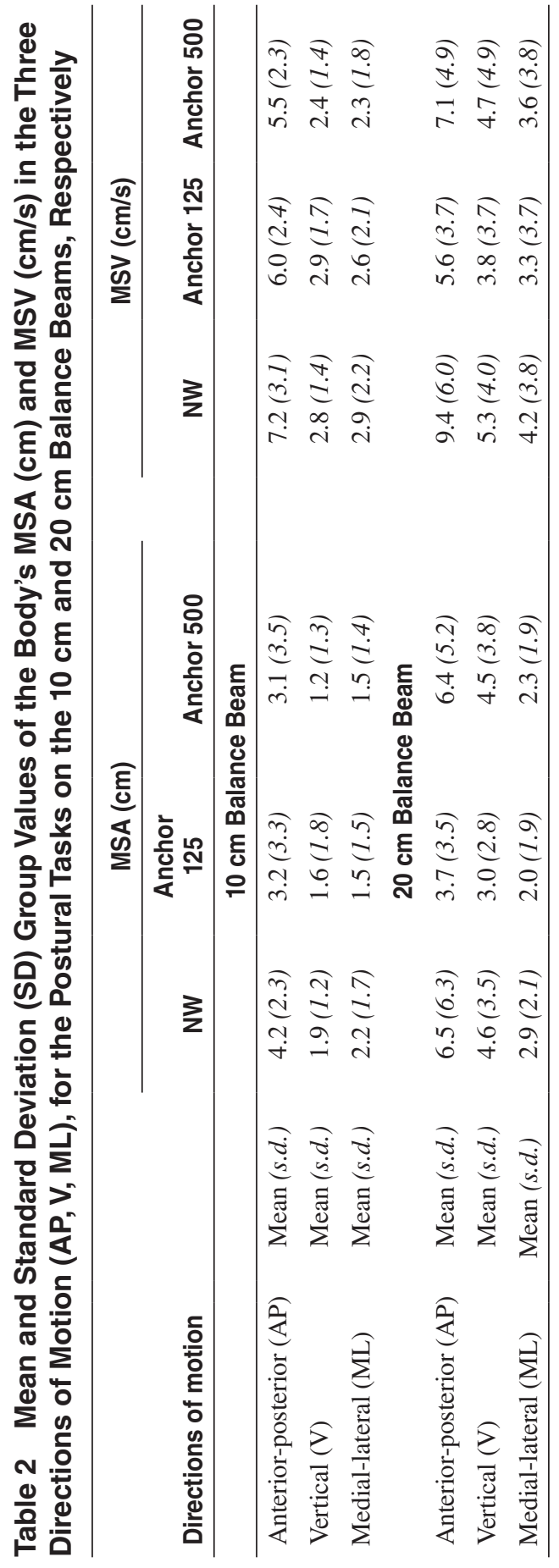


$\mathrm{AP}$ and $\mathrm{V}$ directions indicated that it was higher for the $20 \mathrm{~cm}$ beam than for the $10 \mathrm{~cm}$ beam (Table 3). ANOVA for the ML direction did not exhibit a main effect for beam height, $F(1,10)=4.062, p=0.072$ and for postural task condition, $F(2$, $20)=2.412, p=0.115$, nor for the interaction between beam height and postural task condition, $F(2,20)=0.147, p=0.864$.

For PV in the AP direction, ANOVA identified no main or interaction effects: beam height, $\mathrm{F}(1,10)=2.292, p=.161$; postural task condition, $F(2,20)=1.242$, $p=0.310$; beam height by postural task condition, $F(2,20)=0.311, p=0.736$. In the $\mathrm{V}$ direction, there was a main effect for beam height, $F(1,10)=6.478, p=$ $0.029, \eta^{2}=0.393$ but no main effect for postural task condition, $F(2,20)=2.693$, $p=0.092$ or interaction effect between beam height and postural task condition, $F(2,20)=2.453, p=0.111$. For the ML direction, the ANOVA exhibited the same result found in the $\mathrm{V}$ direction: beam height, $F(1,10)=6.209, p=0.032, \eta^{2}=$ 0.383 ; postural task condition, $F(2,20)=1.879, p=0.179$; beam height by postural task condition, $F(2,20)=0.806, p=0.461$. For both $\mathrm{V}$ and ML directions, PV was higher for the $20 \mathrm{~cm}$ beam than for the $10 \mathrm{~cm}$ beam in both directions.

\section{Path Length}

ANOVA for path length showed a main effect for postural task conditions, $F(2$, $20)=6.388, p=0.007, \eta^{2}=0.390$, but no main effect for beam height, $F(1,10)=$ 2.283, $p=0.162$ and no interaction between beam height and postural task condition, $F(2,20)=1.408, p=0.268$. Post hoc analysis indicated that path length was greater for NW than for the $125 \mathrm{~g}$ anchor weight $(p=0.027)$ but similar to that of the $500 \mathrm{~g}$ anchor weight (Figure 2).

\section{Individual Differences}

Anchoring seemed to improve stability during the blindfolded task for most of the ID participants, although they varied widely across conditions in amplitudes of body oscillation, especially while performing the task on the higher balance beam (i.e., $20 \mathrm{~cm}$ ). They were less affected by the task conditions on the lower balance beam. To illustrate these differences, we selected individuals with varied levels of ID to point out discrepancies in their COM trajectories in both anchor condition tasks as compared with the NW task on the higher balance beam.

The three pairs of examples at the top of Figure 3 are from individuals with mild ID. The first set is from participant CAS, who shows high variability along the task duration for the NW task condition. On the contrary, in both anchor conditions, $125 \mathrm{~g}$ and $500 \mathrm{~g}$, the trajectories remain stable across time. The second set (participant SIL) shows no remarkable variation in any of the task conditions. The third participant (JCO) shows spurts of high variability at the beginning and at the end of the task for the NW condition, and at the end of the tasks for both the $125 \mathrm{~g}$ and $500 \mathrm{~g}$ weight conditions. For this participant, for most of the trajectories' durations, the anchors provided higher stability as compared with the NW condition. The last two sets of examples include individuals with moderate ID. The first, participant LUC, shows high variability in the trajectories, regardless of task condition. The last, participant PAI, with moderate ID and the only Down syndrome participant, shows stable performance during most of the trajectories, except in the beginning of the NW condition, and at the end of the $125 \mathrm{~g}$ weight condition. Both spurts indicate abrupt loss of balance. 


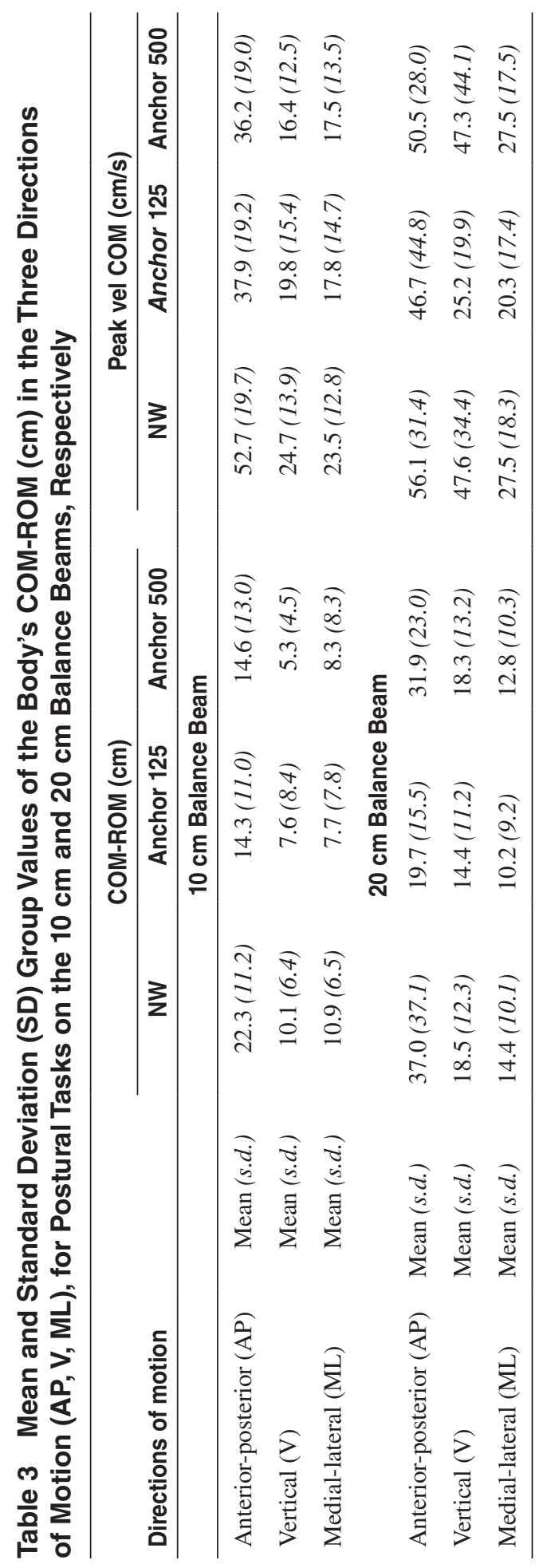




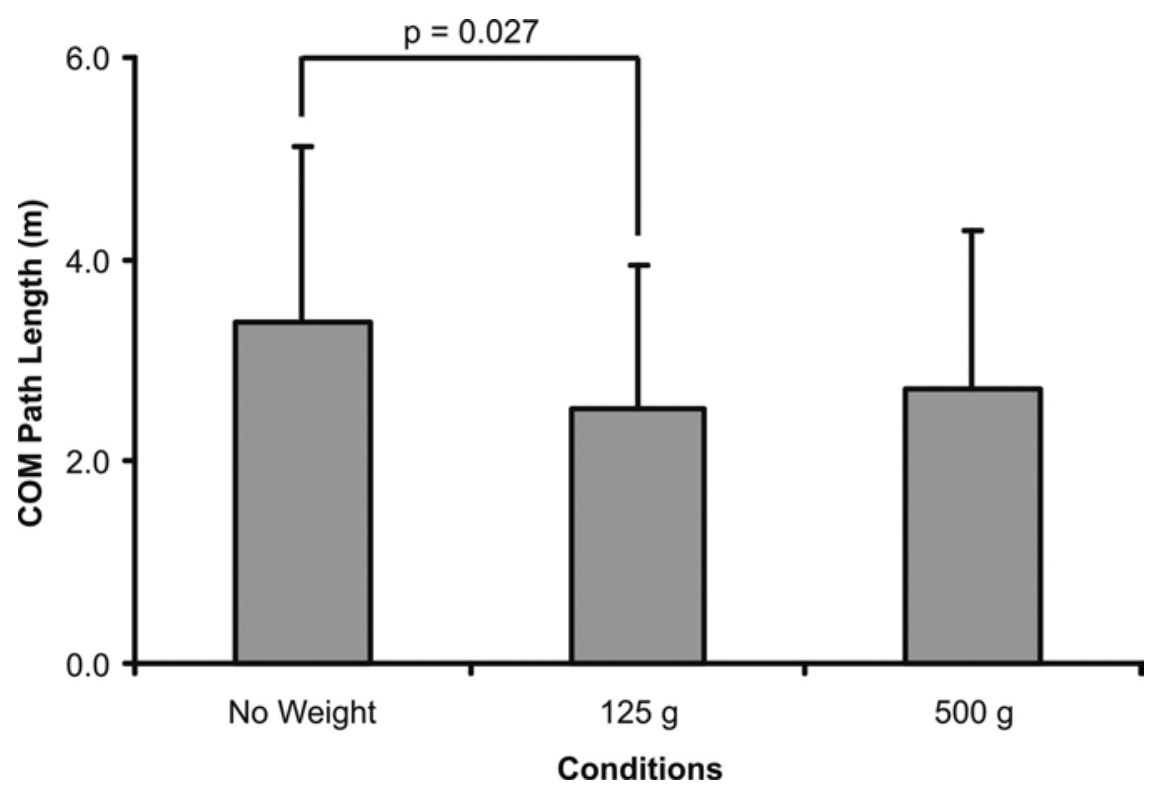

Figure 2 - Mean and standard deviation $(N=11)$ of the COM path length for the three postural task conditions, collapsed across balance beam heights.

\section{Discussion}

We explored how haptic perception contributes to the basic functioning of postural control during individuals' performance of a restricted balance task. In particular, we assessed the abilities of individuals with ID-who are known for postural instability - to use a nonrigid tool (i.e., an "anchor system") to control the forces acting on their bodies, reducing instability when performing restrictive postural tasks, especially when vision is occluded.

Our first prediction was that through the testing of individuals with intellectual disabilities, we would discover that the quality of cognitive development has little impact on how they use control strategies that rely on haptic senses to resolve challenging postural tasks. The second prediction was that increased challenge in the postural task would result in better use of the anchor system, reflected in an improvement in postural stability in contrast with the baseline task condition. In addition, we predicted that the different loads of the anchors would have similar effect on postural stability, and, therefore, haptic sensitivity would be of more significance for orienting the body than would the mechanical support provided by the anchor loads.

Although the group was highly variable in its balance performance, the anchor system appears to have improved the ID individuals' balance over the course of the postural task. This was true especially when participants were naturally unstable or 


\section{Anchor $-125 \mathrm{~g} \longrightarrow$ No Weight -..- Anchor $-500 \mathrm{~g}$}
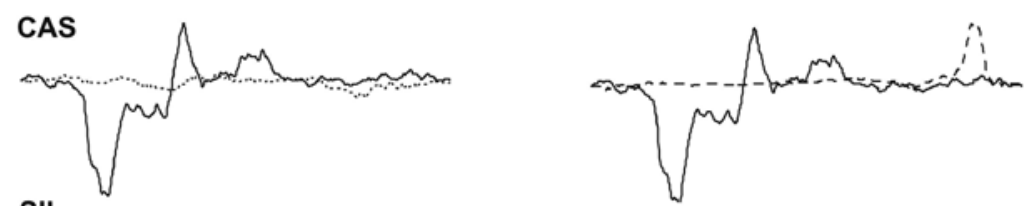

SIL
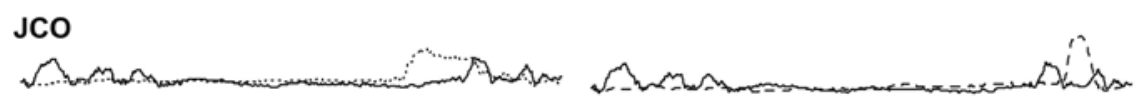

\section{LUC}

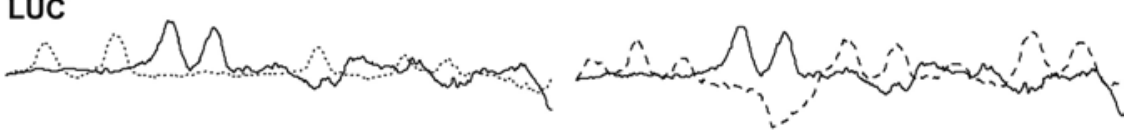

\section{PAI}

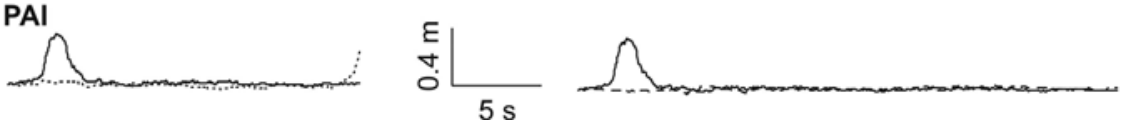

Figure 3 - Individual time series of the COM in the AP direction for the $20 \mathrm{~cm}$ balance beam. NW task condition is contrasted with the $125 \mathrm{~g}$ anchor weight condition (left side) and the $500 \mathrm{~g}$ anchor weight condition (right side) for the following participants: CAS (mild ID), SIL (mild ID), JCO (mild ID), LUC (moderate ID), and PAI (Down syndrome; moderate ID), respectively.

already had poor balance. In contrast to the baseline condition (NW), the reduction in the amount of sway across one of the anchor conditions leads us to believe that a nonrigid tool such as the anchor system can play an important role in facilitating exploratory actions that employ haptic perception.

Compared with the medial-lateral direction, performances in the anteriorposterior direction were less stable due to the positioning of the feet on the balance beam. As expected, the $20 \mathrm{~cm}$-height beam created a less stable support surface than did the $10 \mathrm{~cm}$-height beam. Even though participants performed both beam condition tasks while blindfolded, they were more affected by the higher balance beam, as was detected in most of the tested variables. The brief kinesthetic recall of the act of stepping up on the higher beam seemed to be enough to interfere with attention levels throughout the postural task. Even though individuals were blindfolded, this event added complexity to the task, making it more challenging. Therefore, fear of falling or the expectation of a greater challenge appears to be embedded within the haptic sense' mechanism. 
Overall, an absence of differences between the NW and the $500 \mathrm{~g}$ anchor conditions suggests that during the manipulation of the anchors, the heavier anchor might have increased postural instability as a result of its greater mechanical resistance. In addition, it is possible that the more subtle mechanical resistance of the $125 \mathrm{~g}$ anchor demanded finer control during manipulation; therefore, perturbation had less impact than did that which was generated by the pulling forces in the 500 $\mathrm{g}$ condition. The high variability of this group, however, suggests that prudence should be exercised regarding this interpretation. Indeed, the medium-size effect suggests that for most variables, the use of anchor accounted for $30-40 \%$ of the group variability. The remaining percentage of variance is due to unknown factors.

A movement can be described by its dynamic relationships with systems outside of the organism. In our case, during the exploratory movement the anchor system became a subsystem of the organism; that is, it became subservient to the body's exploratory needs during the postural control effort. The intrinsic perturbation that the absence of vision (the blindfold) caused to the stability state "pushed" the biological system to cooperate with an external system (i.e., the anchor system).

The intellectual condition of the group seemed not to be a constraint to the quality of strategies that individuals in this group used. The need for stability-reached through exploratory actions - is not crucially associated with level of intelligence. Control strategies, which ensure behavioral solutions, also illustrate adaptation. Therefore, just as was found in previous studies (Calve \& Mauerberg-deCastro, 2005; Mauerberg-deCastro, 2003, 2004), here we also corroborated the robust effect of the anchor system with individuals known to have balance problems.

Another study that used the anchor system with nondisabled participants revealed a significant transference in performance during the final application of nonanchor conditions. This was achieved in earlier trials through the manipulation of anchor conditions (Mauerberg-deCastro et al., 2003). Individuals in a control group who performed only repetitive postural tasks, but without the anchors, did not benefit from such transference and exhibited similar levels of instability across task trials. Similar results were found in another study by Mauerberg-deCastro et al. (2006) that employed the anchor paradigm with individuals with ID. In this study anchor weight was varied (i.e., $250 \mathrm{~g}, 500 \mathrm{~g}$, and $1 \mathrm{~kg}$ ), and the baseline condition was tested before and immediately after testing the anchor conditions. The results showed that, as compared with the initial control task, the anchor tasks had a positive effect on postural control, but not when compared with the condition in which the control task was administered at the end of the anchor trials. This suggests a transference effect facilitated by the manipulation of the anchors.

When a participant haptically uses the anchors at various and specific moments during the 30-s period postural task, he or she performs a dual task, even though these are not independent actions. The act of holding the anchors poses a challenge because the individual is required to calibrate the amount of pulling forces to reset their body orientation, which is continuously challenged while standing on a restrictive support surface. We assume that when participants haptically use these anchors, they perform a supra-postural task (Riley, Stoffregen, Grocki, \& Turvey, 1999), and, therefore, posture can both subserve and rely upon a higher level system. It remains an open question as to whether the "anchoring" is due solely to 
the exploratory action itself (i.e., the active seeking out of postural solutions, with the utilization of the anchors as an aid), or to the task constraint (i.e., to keep the anchor resting on the floor, participants had to maintain relatively stable posture), or to a combination of both.

In the studies following the light touch paradigm (Barela, Jeka, \& Clark, 1999; Jeka \& Lackner, 1994; Lackner, Rabin, \& DiZio, 2001)—whether experimental conditions included contact with a rigid or nonrigid surface, the touch force was always applied in a descending direction (i.e., push forces). In our study, we required individuals to "pull away from the surface" with a nonrigid tool. The task instruction was done in such a way that individuals maintained sufficient pull force so that the anchor load was always in contact with, and supported by, the surface. Although we did not measure them, we could observe that there were always pull forces on the ropes as the individuals stretched the anchor extensions.

The detection of distal information provided by the anchor mechanism can be illustrated by Carello et al.'s (2008) conception of the haptic substrate. According to these authors, the haptic substrate relies on the musculoskeletal system as a biotensegrity structure. This refers to the ongoing tension-bearing elements that act on the "network of tightly interconnected soft tissue (muscles, ligaments, and fascia) coupled with external and internal force environments" (p. 343). These tension-bearing structures allow "local activities occurring at multiple parts of the structure naturally to coordinate, therefore re-establishing force balance disturbed by mechanical forces within and adjacent to the system. Therefore, a force applied locally can potentially be sensed globally" (p. 343).

\section{Final Considerations}

The study of haptics has important implications in relation to our knowledge about the process of perception. Its study can also add to our understanding of the phenomenon of the nervous system's dynamic detection of physical invariants (i.e., torque and motion) and the body's use of tools and other nonbiological implements (Turvey, 1996).

When individuals explore their surroundings with rigid tools (canes and ski poles, for example), they gain a significant amount of informational details to improve stability and expand the body's choices for making safe adjustments. Stabilization of body sway can also be achieved through the touch of nonrigid surfaces; during the performance of postural tasks, there is a constant negotiation between postural states and the fluctuating, continuously-changing, dynamic information that nonrigid surfaces provide.

Good postural control is necessary in order for individuals to successfully explore their environments. While exploration results in the detection of information about the world, the quality of this function depends on many constraint factors affecting the organism-including its level of development and, also, on the surrounding environment.

We concluded that for individuals with intellectual disabilities, who historically exhibit poor balance skills during the acquisition of information to enhance postural stability, nonrigid tools play an important role in those exploratory actions that employ haptic perception. It appears that although these individuals are easily 
challenged by postural task demands, regardless of their cognitive skills, they seem to adapt rapidly by improving their postural control via haptic information, which is provided through such nonrigid tools as the "anchor system."

Finally, since the light anchor weight improved the individuals' balance, we concluded that haptic sensitivity was more significant to the orientation of their bodies than was the anchor's mechanical support alone. Mechanical support typically occurs with ongoing resistances of forces greater than $0.4 \mathrm{~N}$ (i.e., ?40 g; Rabin, Dizio, \& Lackner, 2006). While we did not measure exerted forces at the end of each anchor condition, we observed that in order for participants to maintain contact between the $125 \mathrm{~g}$ anchor and the floor, their pull forces logically would have to be less than $125 \mathrm{~g}$. The fact that in some cases their balance was more disrupted by the $500 \mathrm{~g}$ anchor than the $125 \mathrm{~g}$ anchor suggests that they did not depend on mechanical pull. Another possibility, of course, is an intrinsic disruption, caused by excessive pull on the heavier anchor, which reflected on postural stability. Previous studies with nondisabled individuals found no difference among anchor weight conditions (e.g., $125 \mathrm{~g}, 250 \mathrm{~g}, 500 \mathrm{~g}$, and $1 \mathrm{~kg}$; Mauerberg-deCastro, 2004; Mauerberg-deCastro, Viveiros, \& Cozzani, 2005). Intellectual levels do not preclude the efficient use of nonrigid tools to orient body alignment in postural tasks. Therefore, using this experimental paradigm, the ability of individuals with ID to integrate haptic information with motor performance corroborates results found in groups without disabilities.

\section{Recommendations}

As our previous findings indicate, individuals with ID can improve their stability - and hence, opportunities for explorations in postural control-by manipulating an anchor system. The anchor analogy could be used to help explain relationships between action-perception phenomena that occur during the achievement of postural control tasks and related exploration activities. Therefore, task manipulations under specific organism constraints_-such as in the case of intellectual disability-could exploit the extent to which such tools can help stabilize posture through haptic communication. In addition, by investigating different conditions of disability known to be associated with balance problems, we might better understand the limitations of, as well as broader uses of, such tools to enhance postural stabilization. Because the anchor system can facilitate the observation of perceptual-action mechanisms, such a model has practical and theoretical implications. If, in fact, an individual can improve posture and the performance of exploratory tasks by manipulating an anchor as described above, such a model could help provide therapeutic options for individuals who have limitations and impairments in postural control and mobility. For future studies, researchers could exploit such therapeutic effects by inserting practice protocols that use such tools and observe whether there is a transfer effect when compared with balance tasks that do not include these protocols.

\section{Limitations of This Study}

A limitation of this study is that generalizations about individuals with ID and postural behavior should be made with caution. In the current study, we did not 
use a control group, and, therefore, its outcomes can only be compared indirectly with previous studies. Another limitation concerns the small number of participants and varied ID levels. Expanding the number of participants would allow a better understanding of the relationships between levels of ID severity and postural control variations. Finally, another limitation relates to an absence of data acquisition relative to force patterns of the anchor system itself and that are associated with the handling of the anchor extensions. Future studies that employ strain gages attached to the anchor extension, for example, might help us explore anchor tension-bearing loads as control variables in the assessment of coordinative modes and postural sway.

\section{Notes}

1. Haptic sensitivity is the result of coordinative efforts between kinesthetic and tactual abilities that provide awareness of the body, its motion or its orientation in space, and the environment's structure and layout by means of the body itself (i.e., by means of mechanical contact). Carello, Silva, Kinsella-Shaw, and Turvey (2008) defined three different forms of mechanical contact or touch: cutaneous, haptic, and dynamic touch. Cutaneous touch occurs when objects contact the skin, and haptic touch when the hands move over surfaces. Dynamic touch refers to an individual's awareness of magnitudes and directions of movement by implements and limbs through means of muscular effort.

2. The "anchor system" works thus. An individual holds a pair of "anchors," one in each hand (each anchor consists of a rope, with a weight attached at the opposite end, which rests on the floor), under a variety of weight conditions (e.g., $125 \mathrm{~g}, 250 \mathrm{~g}, 500 \mathrm{~g}$, and so forth), while attempting to maintain balance. The anchor system provides a means for the individual to actively seek stability through exploratory motions with his or her hands and arms and to subsequently reorient the entire body in an upright position.

\section{Acknowledgments}

This study was supported by the Brazilian research agency, CNPq/Pibic program, FAPESP, and PROEX/UNESP. We wish to thank the staff and directors of APAE, Rio Claro, Brazil, the participants with intellectual disabilities who volunteered for this study, and their families. Finally, we would like to thank the anonymous reviewers, as well as the $A P A Q$ associate editor, for their insightful review of this paper.

\section{References}

Barela, J.A., Jeka, J.J., \& Clark, J.E. (1999). The use of somatosensory information during the acquisition of independent upright stance. Infant Behavior and Development, 22, $87-102$.

Barela, J.A., Jeka, J.J., \& Clark, J.E. (2003). Postural control in children coupling to dynamic somatosensory information. Experimental Brain Research, 150, 434-442.

Barros, R.M.L., Brenzikofer, R., Baumann, W., Lima, E., Cunha, S., \& Figueroa, P. (1997). A "flexible" computational environment to track markers and reconstruct trajectories in biomechanics. Proceedings of the 15th Congress of the International Society of Biomechanics, Tokyo.

Burton, G. (1993). Non-neural extensions of haptic sensitivity. Ecological Psychology, 5, $105-124$. 
Calve, T., \& Mauerberg-deCastro, E. (2005). Contribuição da percepção háptica no controle postural de crianças. Motriz, 11, 199-204.

Carello, C., Silva, P.L., Kinsella-Shaw, J.M., \& Turvey, M.T. (2008). Muscle-based perception: theory, research and implications for rehabilitation. Revista Brasileira de Fisioterapia, 12, 339-350.

Carvalho, R.L., \& Almeida, G.L. (2009). Assessment of postural adjustments in persons with intellectual disability during balance on the seesaw. Journal of Intellectual Disability Research, 6 [Epub ahead of print].

Cooper, M.M., Carello, C., \& Turvey, M.T. (2000). Perceptual independence of whole length, partial length, and hand position in wielding a rod. Journal of Experimental Psychology. Human Perception and Performance, 26, 74-85.

Easton, R.D., Greene, A.J., DiZio, P., \& Lackner, J.R. (1998). Auditory cues for orientation and postural control in sighted and congenitally blind people. Experimental Brain Research, 118, 541-550.

Gomes, M.M., \& Barela, J.A. (2007). Postural control in Down syndrome: The use of somatosensory and visual information to attenuate body sway. Motor Control, 11, 224-234.

Hale, L., Bray, A., \& Littmann, A. (2007). Assessing the balance capabilities of people with profound intellectual disabilities who have experienced a fall. Journal of Intellectual Disability Research, 51, 260-268.

Holden, M., Ventura, J., \& Lackner, J.R. (1994). Stabilization of posture by precision contact of the index finger. Journal of Vestibular Research, 4, 285-301.

Jeka, J.J., Easton, R.D., Bentzen, B.L., \& Lackner, J.R. (1996). Haptic cues for orientation and postural control in sighted and blind individuals. Perception \& Psychophysics, $58,409-423$.

Jeka, J.J., \& Lackner, J.R. (1994). Fingertip contact influences human postural control. Experimental Brain Research, 100, 495-502.

Jeka, J.J., Schöner, G.S., Dijkstra, T.M.H., Ribeiro, P., \& Lackner, J.R. (1997). Coupling of fingertip somatosensory information to head and body sway. Experimental Brain Research, 113, 475-483.

Johannsen, L., Wing, A.M., \& Hatzitaki, V. (2007). Effects of maintaining touch contact on predictive and reactive balance. Journal of Neurophysiology, 97, 2686-2695.

Krishnamoorthy, V., Slijper, H., \& Latash, M.L. (2002). Effects of different types of light touch on postural sway. Experimental Brain Research, 147, 71-79.

Lackner, J.R., \& DiZio, P. (2005). Vestibular, proprioceptive, and haptic Contributions to spatial orientation. Annual Review of Psychology, 56, 115-147.

Lackner, J.R., Rabin, E., \& DiZio, P. (2001). Stabilization of posture by precision touch of the index finger with rigid and flexible filaments. Experimental Brain Research, 139, 454-464.

Lahtinen, U., Rintala, P., \& Malin, A. (2007). Physical performance of individuals with intellectual disability: A 30 year follow up. Adapted Physical Activity Quarterly, 24, $125-143$.

Mauerberg-deCastro, E. (2003). Expansão da sensibilidade háptica através de um sistema "âncora" durante o andar sob restrição visual e de equilíbrio [Expanding haptic sensitivity through an "anchor" system during walking, using visual and balance constraints]. Relatório de pesquisa CAPES [Technical research grant report, CAPES] Brasil.

Mauerberg-deCastro, E. (2004). Developing an anchor system to enhance postural control. Motor Control, 8, 339-358.

Mauerberg-deCastro, E., \& Angulo-Kinzler, R. (2000). Locomotor patterns of individuals with Down syndrome: Effects of environmental and task constraints. In D. Elliot, R. Chua \& D. Weeks (Eds.) Perceptual-motor behavior in Down syndrome (pp. 71-98). Champaign, IL: Human Kinetics. 
Mauerberg-deCastro, E., Calve, T., Viveiros, F.F., Polanczyk, S., \& Cozzani, M.V. (2003). Um Tutorial Sobre Percepção Háptica no Controle Postural: Ilustrando um Sistema "âncora" e suas Aplicações na Reabilitação e na Atividade Física Adaptada [A tutorial about haptic perception and postural control: Illustrating an "anchor" system and its application in rehabilitation and adapted physical activity]. Revista da Sociedade Brasileira de Atividade Motora Adaptada, 8, 7-20.

Mauerberg-deCastro, E., Viveiros, F.F., \& Cozzani, M.V. (2005). The use of non-rigid tools by adults with mental retardation to control posture. Proceedings Book of the International Symposium of Adapted Physical Activity. Verona. 54-54.

Mauerberg-deCastro, E., Viveiros, F.F., Cozzani, M.V., Moraes, R., Cuba, B.W., \& Silva, E.M. (2006). The use of an "anchor system" to improve stability during balance tasks by individuals with mental retardation. Proceedings from 2006 North American Federation of Adapted Physical Activity (NAFAPA) Symposium. Ann Arbor. 47-47.

Prioli, A.C., Cardozo, A.S., Freitas Junior, P.B., \& Barela, J.A. (2006). Task demand effects on postural control in older adults. Human Movement Science, 25, 435-446.

Rabin, E., DiZio, P., \& Lackner, J.R. (2006). Time course of haptic stabilization of posture. Experimental Brain Research, 170, 122-126.

Rabin, E., DiZio, P., Ventura, J., \& Lackner, J.R. (2008). Influences of arm proprioception and degrees of freedom on postural control with light touch feedback. Journal of Neurophysiology, 99, 595-604.

Riley, M.A., Stoffregen, T.A., Grocki, M.J., \& Turvey, M.T. (1999). Postural stabilization for the control of touching. Human Movement Science, 18, 795-817.

Shumway-Cook, A., \& Woollacott, M. (1985). The growth of stability: postural control from a developmental perspective. Journal of Motor Behavior, 17, 131-147.

Solomon, H.Y., \& Turvey, M.T. (1988). Haptically perceiving the distances reachable with hand-held objects. Journal of Experimental Psychology. Human Perception and Performance, 14, 404-427.

Thalheimer, W. \& Cook, S. (2002, August). How to calculate effect sizes from published research articles: A simplified methodology. [Retrieved February 1, 2009 from http:// work-learning.com/effect_sizes.htm]

Turvey, M.T. (1996). Dynamic touch. The American Psychologist, 51, 1134-1152.

Vuillerme, N., Isableu, B., \& Nougier, V. (2006). Attentional demands associated with the use of a light fingertip touch for postural control during quiet standing. Experimental Brain Research, 169, 232-236.

Winter, D.A. (2005). Biomechanics and Motor Control of Human Movement (3rd ed.). New York: Wiley. 
Copyright of Adapted Physical Activity Quarterly is the property of Human Kinetics Publishers, Inc. and its content may not be copied or emailed to multiple sites or posted to a listserv without the copyright holder's express written permission. However, users may print, download, or email articles for individual use. 
Copyright of Adapted Physical Activity Quarterly is the property of Human Kinetics Publishers, Inc. and its content may not be copied or emailed to multiple sites or posted to a listserv without the copyright holder's express written permission. However, users may print, download, or email articles for individual use. 